\title{
Reconstruction of Plantar Forefoot in Diabetic Foot Ulcers: A Comparative Study of Perforator Flaps and Random Flaps
}

\author{
Yun-Seob Kim (D), Si-Gyun Roh (D), Jong-Lim Kim (D), Nae-Ho Lee (D), Jin Yong Shin (D) \\ Department of Plastic and Reconstructive Surgery, Jeonbuk National University Hospital, Jeonju, Korea
}

\begin{abstract}
Background: Plastic and reconstructive surgeons consider a variety of options to salvage limbs of patients when operating on soft tissue defects of the plantar forefoot in diabetic foot ulcers. This study focuses on the efficacy and durability of perforator flaps compared to that of random flaps, when covering the plantar forefoot area in diabetic patients.

Methods: From January 2011 to August 2020, we performed 49 plantar forefoot reconstructions to cover soft tissue defects in diabetes patients using local random flaps, regional perforator-based flaps, or free flaps. The patients' clinical characteristics, size of defects, surgical outcomes and complications were reviewed retrospectively.

Results: Forty-nine patients underwent plantar forefoot reconstruction over the period reviewed. Eleven patients were female and the others were male. The mean age of the patients was 54.2 years (range, 32-73 years). Median follow-up was 5.2 months. The mean size of the defect was $7.2 \times 4.5 \mathrm{~cm}$. During follow-up periods, partial or complete loss of flaps was closely observed. We identified wound complications in $67 \%$ of local random flaps compared to $11 \%$ and $21 \%$ in regional and free flaps, respectively.

Conclusion: Soft tissue defects in the distal third of the foot can severely worsen diabetic patients' quality of life. Perforator-based flaps, providing better vascularized tissue than random flaps, can be used to attain acceptable functional and aesthetic results for plantar forefoot reconstruction in diabetes patients.
\end{abstract}

Keywords: Diabetic foot ulcer; Reconstructive surgery; Perforator flap

\section{Introduction}

According to a study recently conducted in South Korea, the number of patients suffering from diabetes mellitus-related foot ulcers amounts to approximately $1.4 \%$ of the entire population, with new patients accounting for $0.4 \%$ of the entire population emerging each year. The expenses for treating the patients reached USD 460 million in 2016 [1].

As the plantar forefoot bears the weight of the body and is critical for ambulation, reconstructing for recovery of function as well as appearance is highly valued. However, the reconstruction procedure itself is not easy because of the complex vascularity of the forefoot. Further, the planter forefoot frequently involves complications of peripheral arterial disease in diabetes mellitus patients [2,3].

Reconstructive operation of plantar forefoot often employs local flaps, regional flaps, or free flaps. In this study, the authors used local random flaps, regional perforator-based flaps, or free flaps as reconstructive options in diabetic plantar forefoot lesions. We attempted to clarify the difference between random flaps and perforator flaps in terms of flap durability and efficacy.

\section{Original Article}

Received: July 16, 2020

Revised: November 6, 2020

Accepted: November 7, 2020

\section{Corresponding author:}

Si-Gyun Roh, M.D.

Department of Plastic and Reconstructive Surgery, Jeonbuk National University Hospital, 20 Geonji-

ro, Deokjin-gu, Jeonju 54907, Korea

Tel: +82-63-250-1374

Fax: +82-63-250-1866

E-mail: pssroh@jbnu.ac.kr

This is an Open Access article distributed under the terms of the Creative Commons Attribution Non-Commercial License (https://creativecommons.org/licenses/by-nc/4.0/) which permits unrestricted non-commercial use, distribution, and reproduction in any medium, provided the original work is properly cited.

C) 2021 Korean Wound Management Society 


\section{Methods}

The medical records over the period from January 2011 to August 2020 of 49 patients with defects due to diabetes mellitus in the plantar forefoot were the subject of this retrospective study. Two cases among the patients had a previous history of reconstruction at other hospitals employing different flap procedures on the same site. All patients provided written informed consent for the publication and the use of their images.

\section{Preoperative evaluation}

The preoperative arterial patency of lower extremities was identified through computed tomography scan or Doppler ultrasound, and in cases where stenotic lesions occupied more than $50 \%$ of the arterial lumen, an appropriate level of patency was secured through a cardiologist's intravascular intervention. Afterwards, the arterial patency of lower extremities was re-evaluated with post-interventional ankle-brachial index and physical examination, checking for foot warmth and relief of infection. Further, the presence and positions of perforators, and locations of arteries, were found by using the handheld Doppler device. The presence of inflammation in lesions was also appraised in preoperative wound cultures and blood tests; the patients then underwent operation with either mild inflammations (i.e., C-reactive protein $<10 \mathrm{mg} / \mathrm{L}$ ) or no inflammation [4].

\section{Surgical technique}

First of all, the patients were anesthetized, and their recipient sites were properly cleaned and debrided, after which the size of the recipient site defect was remeasured. Different flap procedures were employed depending on the size of defects: for defects less than $4 \mathrm{~cm}^{2}$, between 4 and $20 \mathrm{~cm}^{2}$, and over 20 $\mathrm{cm}^{2}$; local random flaps, regional flaps, and free flaps were applied respectively. After deciding the type of flap to be applied, for regional and free flaps, the perforator vessels were found with an intraoperative handheld Doppler device, and then the flap was designed.

For cases where local random flaps were applied, local advancement flaps, V-Y advancement flaps, or rotational flaps were used. For cases with regional flaps, a medial plantar artery perforator base pedicled flap was used in the present study for which a skin graft was applied to the donor site. In cases with free flaps, the anterolateral thigh (ALT) fasciocutaneous free flap, vastus lateralis myocutaneous free flap, or superficial circumflex iliac artery perforator free flap were used, and primary closure was applied to all donor sites. The choice of free flap was determined by the size of the defect, the degree of need for soft tissue padding and the availability of donor sites. Upon completion of the operation, splints were applied to all patients, preventing them from bearing weight for 3-4 weeks. Full weight-bearing was allowed after 5-6 weeks from completion of operation.

\section{Outcomes}

A retrospective chart review of medical records of all patients was conducted, and complications of the wounds were classified into three scales: partial loss, total loss, and recurrence. Cases with no wound complications were excluded from the classification, while cases with partial flap necrosis or onset of dehiscence were classified as "partial loss"; cases with necrosis of the entire flap were defined as "total loss." Cases with recurrent ulceration on the site of flap coverage after discharge were referred to as "recurrence," and cases where ulcers or infections occurred on other sites were not included in this category.

\section{Results}

Of the 49 subjects, 38 were male subjects. The average age of all patients was 54.2 years (range, 32-73 years); the mean follow-up was 5.2 months. Measurement of average defect size was $32.4 \mathrm{~cm}^{2}$ (range, $1-140 \mathrm{~cm}^{2}$ ) (Table 1).

Local flaps were provided to 22 patients in total. The medial plantar artery pedicled flap was applied to nine patients, whereas a free flap was applied to 18 patients. Where the medial plantar flap was applied, the donor site was fully covered with a split-thickness skin graft; where a free flap was applied, all donor sites were primarily closed (Table 2). The two patients who had received flap surgery prior to operation at our hospital were provided with ALT fasciocutaneous free flaps.

During follow-up, total necrosis occurred in one case, which

Table 1. Summary of patients

\begin{tabular}{lc}
\hline Characteristics & Value \\
\hline No. of patients & 49 \\
Age $(\mathrm{yr})$ & $54.2(32-73)$ \\
Sex & \\
Male & 38 \\
Female & 11 \\
Defect size $\left(\mathrm{cm}^{2}\right)$ & $32.4(1-140)$ \\
\hline
\end{tabular}

Values are presented as number or mean (range). 
Table 2. Choice of flap and donor site closure

\begin{tabular}{lc}
\hline Characteristic & No. of patients (\%) \\
\hline Type of flap $(n=49)$ & $22(45)$ \\
Local random flap & $9(18)$ \\
Regional flap & $18(37)$ \\
Free flap & \\
Donor site closure $(n=27)$ & $9(33)$ \\
STSG & $18(67)$ \\
Primary closure & \\
\hline
\end{tabular}

STSG, split-thickness skin graft.

was subsequently treated by secondary healing without implementing revision. Partial necrosis was found in nine patients for which conservative treatment was continued without a second operation. The wounds of all patients with complications were healed completely during follow-up. After completion of wound healing, however, 10 patients experienced recurrence of complications; another flap surgery was provided to nine patients while the other patient was not provided with any additional operation. Specifically, in cases of local flap patients, recurrence occurred at 3-12 weeks after surgery, and in free flap patients, at 8-52 months after surgery. Overall, the complication rate in local random flaps was higher than other flap options (Table 3). In local random flaps, $67 \%$ of patients experienced postoperative wound complications, compared to $11 \%$ in regional flap patients and $21 \%$ in free flap patients.

\section{Discussion}

As discussed above, plantar forefoot reconstruction amounts to a significant challenge for a plastic surgeon, as the site is functionally crucial for ambulation of patients, and unsuccessful reconstruction will also result in much aesthetic damage. Further, defect of this site inevitably affects the economic status of patients, significantly damaging the quality of their lives [5].

For diabetic foot ulcers, the surgical approach is particularly difficult because vascular statuses are likely to be compromised. Nevertheless, recently conducted studies indicate the ratio of successful reconstruction in patients suffering from diabetic foot ulcers is increasing [6,7], while amputation as an alternative has been reported to offer no significant comparative gain [8]. Therefore, reconstructive surgery for plantar forefoot defects of patients suffering from diabetes mellitus is being recognized as an effective measure.
Table 3. Wound complication rate categorized by flap type

\begin{tabular}{lccc}
\hline Flap & Partial loss & Total loss & Recurrence \\
\hline Local random flap & $8(36)$ & 0 & $7(31)$ \\
Regional flap & $1(11)$ & 0 & 0 \\
Free flap & 0 & $1(5)$ & $3(16)$ \\
\hline
\end{tabular}

Values are presented as number (\%).

Conventionally, the relatively simple procedure of applying a skin graft to the plantar forefoot was the preferred method. However, hyperkeratosis, contracture, and frequent recurrence of ulceration makes the skin graft less appropriate for sites that bear weight [9], motivating the authors to conduct this study on flap procedures.

When the defect area is small, local flaps can be used under partial anesthesia in relatively short operations, which is particularly favorable for patients with systemic conditions. In addition, the surgery leaves no donor site morbidity, and also has the advantage of tissue similarity because the defect area can be replaced with adjacent tissue (Fig. 1) [10].

Regional flaps also offer the benefits of tissue similarity (in the present study, ipsilateral medial plantar artery perforator pedicled flaps were used). Moreover, the regional flap can cover larger defects than those covered with local flaps, and unlike the distant free flap, it attains durable and glabrous skin without flap bulkiness (Fig. 2) [11].

Meanwhile, the free flap has the advantage of an even bigger size to cover larger defects, versatility in application regardless of the defect position, and convenient identification of vascularity (Fig. 3) [12].

But regardless of the type of flap procedure, identifying the preoperative statuses of patients is essential. The statuses comprise comorbid conditions, vascular insufficiency, control of infection, and foot deformities, etc. For diabetic foot patients, about $15 \%$ are known to have vascular problems [13]. Flapbased reconstruction in such patients is generally not feasible. However, because flap reconstruction and limb retention show higher 5-year survival rates and better economic implications for patients as compared to amputation, it should be given thorough consideration in determining the best option [14].

In the results, the wound complication rate in local flaps was higher than other flap surgeries. This could be attributed to the high degree of tension around the operation wound and soft tissue padding insufficient to endure the weight borne on the foot.

The shortcomings of the present study are mainly the small group of subjects and insufficient period of follow-up. The fact 

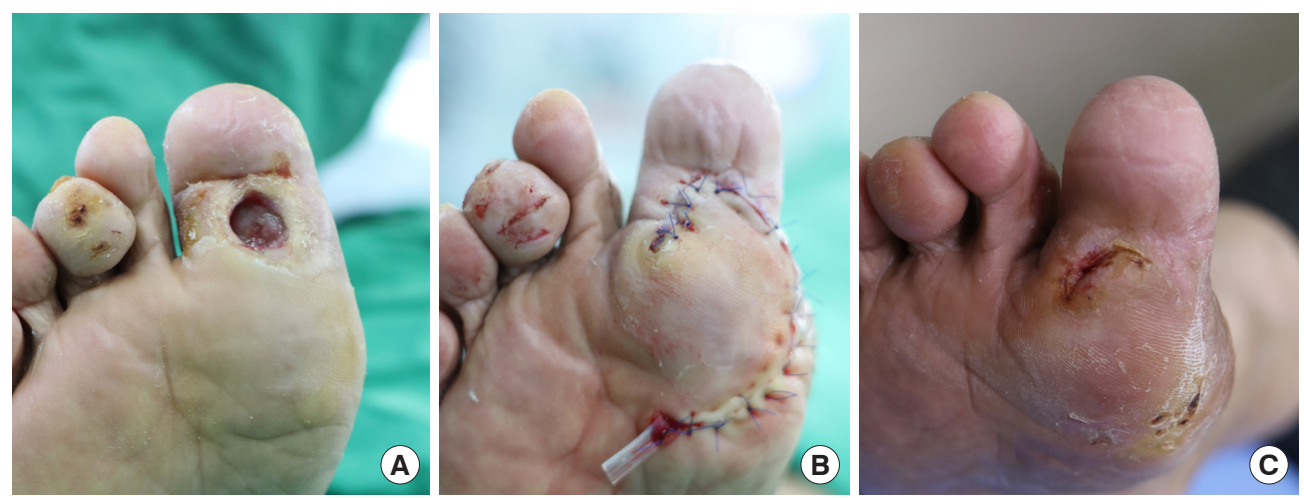

Fig. 1. Local random flap. (A) Preoperative state. (B) Immediate postoperative state. (C) Result at 60th postoperative day.
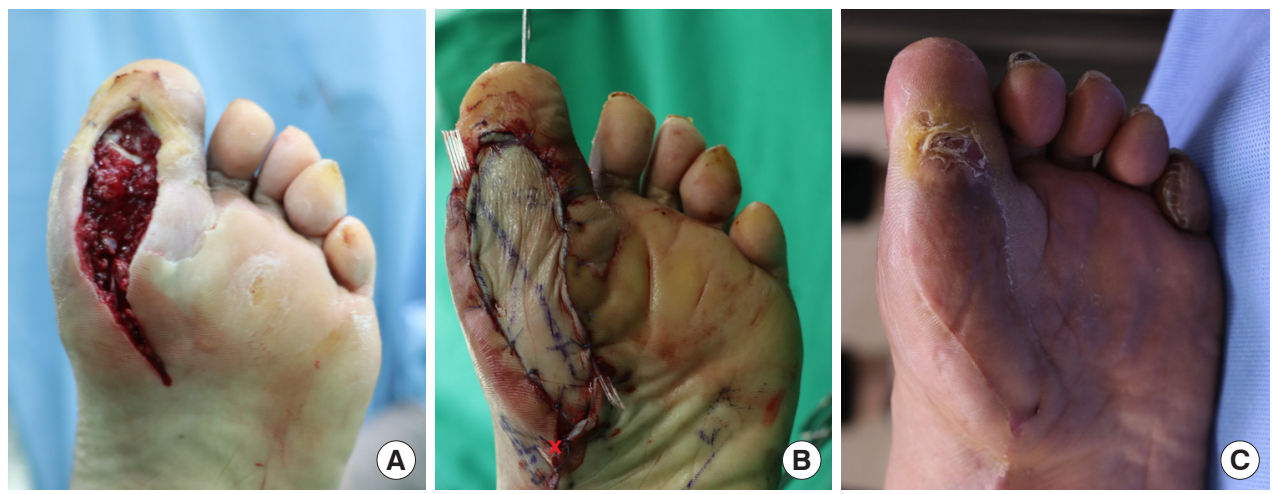

Fig. 2. Medial plantar artery perforator pedicled flap. (A) Preoperative state. (B) Immediate postoperative state. (C) Result at 55th postoperative day.
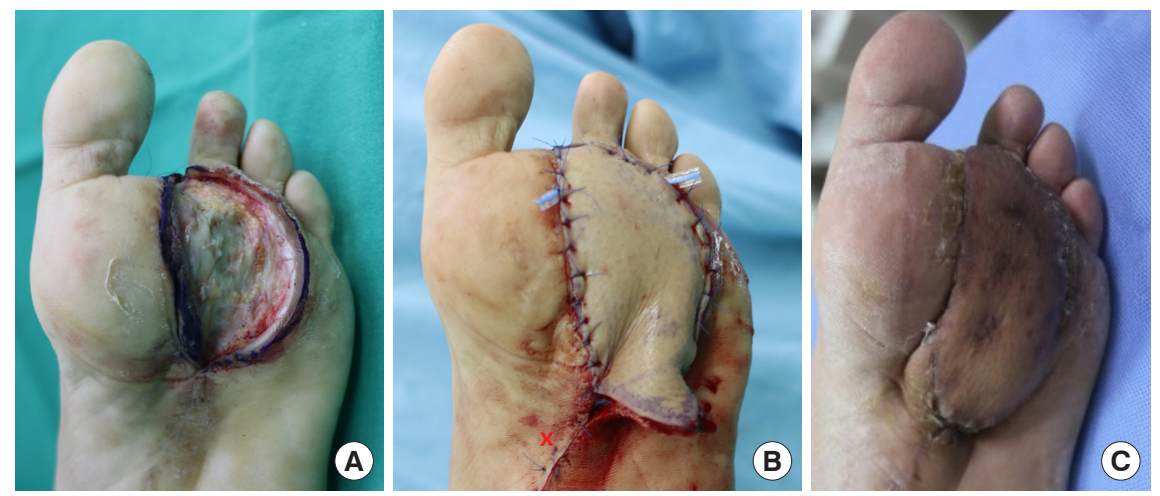

Fig. 3. Anterolateral thigh free flap. (A) Preoperative view. (B) Immediate postoperative view. (C) Result at 65 th postoperative day.

that the recurrences were observed in patients who had longer periods of follow-up warrants more extended research. Furthermore, while it is difficult to control sizes of defects for a particular study, we acknowledge that comparing efficacy across different types of flaps is disputable when the types of flap surgery were determined by the size of the defect.

Nevertheless, based on the total complication rates in each flap type, we contend that for patients suffering plantar fore- foot defects due to diabetes mellitus, the perforator flap procedure can bring about better functional and aesthetic recovery than a random flap.

\section{Conflict of interest}

No potential conflict of interest relevant to this article was reported. 


\section{ORCID iDs}

Yun-Seob Kim

https://orcid.org/0000-0002-4443-6434

Si-Gyun Roh

https://orcid.org/0000-0003-2865-0075

Jong-Lim Kim

https://orcid.org/0000-0002-7110-4574

Nae-Ho Lee

https://orcid.org/0000-0003-1354-8203

Jin Yong Shin

https://orcid.org/0000-0003-2680-7411

\section{References}

1. Chun DI, Kim S, Kim J, et al. Epidemiology and burden of diabetic foot ulcer and peripheral arterial disease in Korea. J Clin Med 2019;8:748.

2. Pallua N, Di Benedetto G, Berger A. Forefoot reconstruction by reversed island flaps in diabetic patients. Plast Reconstr Surg 2000;106:823-7.

3. Yang SL, Zhu LY, Han R, et al. Pathophysiology of peripheral arterial disease in diabetes mellitus. J Diabetes 2017;9: 133-40.

4. Hong JP, Oh TS. An algorithm for limb salvage for diabetic foot ulcers. Clin Plast Surg 2012;39:341-52.

5. Reiber GE, Lipsky BA, Gibbons GW. The burden of diabetic foot ulcers. Am J Surg 1998;176:5S-10S.

6. Moucharafieh RS, Saghieh S, Macari G, et al. Diabetic foot salvage with microsurgical free-tissue transfer. Microsurgery 2003;23:257-61.

7. Karp NS, Kasabian AK, Siebert JW, et al. Microvascular free-flap salvage of the diabetic foot: a 5-year experience. Plast Reconstr Surg 1994;94:834-40.

8. Gibbons GW, Marcaccio EJ Jr, Burgess AM, et al. Improved quality of diabetic foot care, 1984 vs 1990 . Reduced length of stay and costs, insufficient reimbursement. Arch Surg 1993;128:576-81.

9. Lofstrand JG, Lin CH. Reconstruction of defects in the weight-bearing plantar area using the innervated free medial plantar (Instep) Flap. Ann Plast Surg 2018;80:245-51.

10. Ramanujam CL, Zgonis T. Use of local flaps for soft-tissue closure in diabetic foot wounds: a systematic review. Foot Ankle Spec 2019;12:286-93.

11. Scaglioni MF, Rittirsch D, Giovanoli P. Reconstruction of the heel, middle foot sole, and plantar forefoot with the medial plantar artery perforator flap: clinical experience with 28 cases. Plast Reconstr Surg 2018;141:200-8.

12. Hong JP. Reconstruction of the diabetic foot using the anterolateral thigh perforator flap. Plast Reconstr Surg 2006; 117:1599-608.

13. Armstrong DG, Cohen K, Courric S, et al. Diabetic foot ulcers and vascular insufficiency: our population has changed, but our methods have not. J Diabetes Sci Technol 2011;5: 1591-5.

14. Oh TS, Lee HS, Hong JP. Diabetic foot reconstruction using free flaps increases 5-year-survival rate. J Plast Reconstr Aesthet Surg 2013;66:243-50. 